\title{
Cerebral lesions and climbing suppression in the turtle'
}

IRWIN M. SPIGEL AND KENNETH R. ELLIS

UNIVERSITY OF TORONTO

The effects of forebrain lesions on a form of passive avoidance were examined under varying shock intensities. The results suggested that damage to cerebral tissue impaired the climbing response of Chrysemys, independent of shock level, rather than the acquisition of avoidance behavior. The data appeared to confirm the participation of reptile hemispheric tissue in emotionally integrative behavior patterns.

The effects of differentially placed lesions in the mammalian forebrain on active and passive avoidance acquisition have been given increasing study in recent years (see e.g., Lubar, 1964; McCleary, 1961). Interest in the reptile forebrain has been largely confined to the search for structural and functional homologies with mammalian brain tissue. Neurophysiological techniques applied to this problem, however, have produced data that are either incomplete or ambiguous. The inadequacies of earlier study have been reviewed by Goldby \& Gamble (1957).

Though Bremer, Dow, \& Moruzzi (1939) failed to evoke motor responses with cortical stimulation, earlier study, summarized by Goldby and Gamble, had shown spontaneous movement deficit with extensive cerebral ablation in the turtle; and loss of appropriate motor responses during emotional states in snake following such ablation. Despite this evidence of deficit, the electrical inexcitability of the reptile cortex has been taken by Goldby and Gamble to indicate that this tissue is not comparable to the mammalian "motor" area. The failure of Kruger \& Berkowitz (1960) to find any retrograde degeneration in the thalamus following cortical lesions is consistent with this position. Goldby and Gamble further suggested that the basal parts of the hemisphere may be of significance in the orientation of the reptile to its environment-possibly by facilitating neural mechanisms at lower levels and integrating appropriate behavior patterns.

Several experiments in our laboratory confirmed the loss of spontaneous movement with massive cerebral damage in the turtle, but the data clearly showed that less extensive lesions failed to affect either locomotor activity levels or the acquisition of escape in a one-way shuttle-box.

\section{EXPERIMENT 1}

Subjects. Three groups of ten male Chrysemys, three to four years of age as estimated from carapace length, were randomly constituted as lesioned Ss, sham operates and unoperated controls. Three of the sham operates died before the experiment was complete so that the data of only 27 Ss was available.
Apparatus. A detailed description appears elsewhere (Spigel \& Ellis, 1965). The apparatus, essentially, consists of a circular metabolism cage wired for shock administration when Ss, with a mercury switch taped to their carapace, begin to climb. When they reach an approximately $20^{\circ}$ lie, the switch is thrown, sending a current through the $S$ between the cage wall and floor. A timer, also activated, recorded the number of seconds $\mathrm{S}$ actually spent climbing under these conditions. Previous study (Spigel, 1964; Spigel \& Ellis, 1965) had shown that $\mathrm{Ss}$ in such a walled surround are persistant in their efforts to climb out. The suppression of this effort was taken operationally as demonstration of a form of passive avoidance.

Procedure: Surgery. Ss were anesthetized by retention for $75 \mathrm{~min}$. at $0^{\circ} \mathrm{C}$. Mechanically produced bilateral lesions were confined to the posterior and mid-hemispheric regions. Sham operates were frozen in similar fashion and the skull drilled bilaterally without penetration of the dura.

Testing. Behavioral examination began one week after surgery. Ss were placed in the climbing apparatus for $30 \mathrm{~min}$. each day with shock intensity set at 60 volts $\mathrm{AC}$. A criterion of one $30-\mathrm{min}$. session without climbing was established, and both the number of trials to criterion and the number of seconds of climbing each day were recorded.

Results

Since neither the number of trials to criterion, nor the mean climbing time per trial were significantly different for sham operated and unoperated controls, their data were pooled. Mean scores on both measures for lesioned and control Ss are recorded in the top row of Table 1. Mann-Whitney U-tests of data from both measures failed to differentiate the groups.

\begin{tabular}{|c|r|r|r|r|}
\cline { 2 - 5 } \multicolumn{1}{c|}{} & \multicolumn{2}{c|}{$\begin{array}{c}\text { MEAN TRIALS } \\
\text { to ZERO }\end{array}$} & \multicolumn{2}{c|}{$\begin{array}{c}\text { MEAN CLIMBING } \\
\text { per TRIAL(sec's) }\end{array}$} \\
\cline { 2 - 5 } & LESION & CONTROL & LESION & CONTROL \\
\hline 60 VOLT & 4.8 & 3.6 & 4.4 & 3.0 \\
\hline 30 VOLT & 2.9 & 10.1 & 5.2 & 24.4 \\
\hline 0 VOLT & 1.7 & 10.2 & 385.4 & 639.2 \\
\hline
\end{tabular}

Table 1. Scores on Two Measures of Climbing for Lesioned and Control Ss under Varying Shock Intensities 


\section{EXPERIMENT II}

Subjects. Two groups of eight and two groups of six male Chrysemys, of the same age range as in Experiment I were constituted-the former groups assigned to 30-volt lesioned and sham-operated conditions, and the latter to a 0 -volt test situation.

Apparatus, Surgery and Testing were identical to that described for the first experiment. The only procedural difference was the setting of a 30 -volt shock intensity for the lesioned and control Ss of the two groups of eight, and no shock at all for the six Ss in each of the remaining groups.

\section{Results}

The scores for the four groups of Experiment II are contained in the remainder of Table 1 . While the difference in the mean climbing time under the 0 -volt condition was not statistically significant for the lesioned and sham-operated Ss, a two-tailed Mann-Whitney Utest of the difference in the mean number of trials to criterion $(U=10)$ indicated significance at less than the .05 level. For the 30 -volt condition, the sham-operated Ss yielded significantly higher scores on both measures than lesioned Ss $(U=9,8 ; p<.01$, respectively). Additional Mann-Whitney U-tests also indicated a significant difference between the lesioned Ss of the 0 -volt and 30-volt groups $(U=4, p<.05)$ and between the 0 -volt and 30-volt sham-operated controls $(U=0, p<.01)$. The number of trials to criterion was not statistically differentiated across shock intensity groups with respect to climbing time per trial. Typical lesions are shown schematically in Fig. 1.

\section{Discussion}

It appeared from the results of Experiment I that the reduced response levels of all groups under the highe intensity shock might have obscured any lesion-induced effects. It further appeared that an additional set of control conditions, namely climbing behavior in the absence
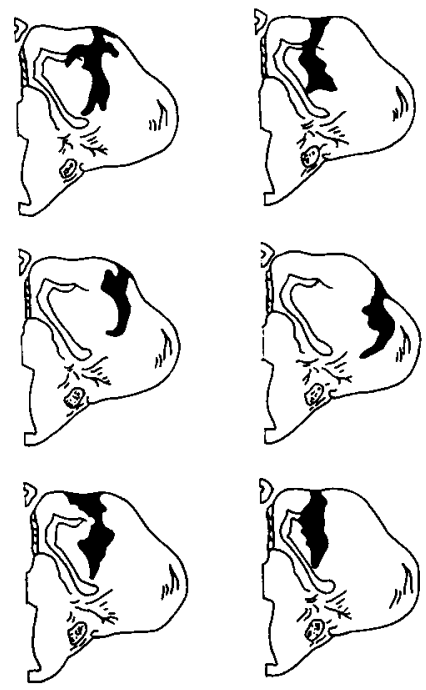

Fig. 1. Typical hemispheric lesions in operated Ss. of shock, might be necessary for a more thorough evaluation of any emerging deficit.

Although significantly increased climbing was observed for sham operates over lesioned Ss under the 30-volt condition, the persistence of this differenceeven without contingent shock-suggested that the lesioned-induced deficit related to climbing behavior per se, rather than to the acquisition of passive avoidance. The inference of a general activity deficit attributable to the less extensive lesions administered was rendered unlikely in view of previous unpublished data which clearly showed that such Ss did not differ at all from controls with respect to locomotion.

Climbing under the conditions currently imposed represented an attempt to escape, and as such apparent$1 y$ involved emotionally integrative mechanisms. An interpretation of the observed climbing deficit in terms of interference with, or inhibition of a response more ubiquitously generated or a roused by confinement seems more appropriate. In this regard, the lesion-induced climbing decrement in a situation which has been shown to be relatively repugnant to this species, is consistent with earlier observation of the loss of appropriate motor responses in the cerebrally-lesioned snake under emotional stimulation. To this extent, the participation of reptile hemispheric tissue in emotionally integrative behavior patterns is suggested.

The data provided by the sham operates also substantiates the results of an earlier study (Spigel \& Ellis, 1965) of climbing suppression in which mean climbing per trial statistically differentiated 0 -volt and 30 -volt $\mathrm{Ss}$, while number of trials to zero did not. While it is tempting to extend the comparison of $0-$ and 30-volt Ss with those under 60-volt shock in Experiment I, the dangers inherent in cross-experimental tests are augmented in the case of turtles by seasonal differences at the time of capture. Nevertheless, the differences between Ss in these groups, as shown in Table 1, are sufficient to warrant further study.

\section{References}

Bremer, F., Dow, S., \& Moruzzi, G. Physiological analysis of the general cortex in reptiles and birds. J. Neurophysiol., 1939, 2, 473-487.

Goldby, F., \& Gamble, H. J. The reptilian cerebral hemispheres. Biol. Rev., 1957, 32, 383-420.

Kruger, Lawrence, \& Berkowitz, E. C. The main afferent connections of the reptilian telencephalon as determined by electrophysiological methods. J. comp. Neurol., 1960, 115, 125-141.

Lubar, J. F. Effect of medial cortical lesions on the avoidance behavior of the cat. J. comp. physiol. Psychol., 1964, 58, 38-46.

McCleary, R. A. Response specificity in the behavioral effects of limbic system lesions in the cat. J. comp. physiol. Psychol., $1961,54,605-613$.

Spigel, I. M. Learning, retention and disruption of detour behavior in the turtle. J. comp. physiol. Psychol., 1964, 57, 108-112. (a)

Spigel, I. M., \& Ellis, K. R. Climbing suppression: Passive avoidance in the turtle. Psychon. Sci., 1965, 3, 215-216.

\section{Note}

1. This research was supported by Grant No. APA-133 from the National Research Council of Canada. 